\title{
Integration of MRI in Evaluation and Ablation of Atrial Fibrillation
}

\author{
RS MacLeod ${ }^{1,2,3,4}$, E Kholmovski ${ }^{5}$, EVR DiBella ${ }^{4,5}$, RS Oakes ${ }^{2}, \mathrm{JE} \mathrm{Blauer}^{1,4}$, E Fish $^{2,4}$, \\ $\mathrm{S}$ Vijayakumar ${ }^{5}, \mathrm{M}$ Daccarett $^{2}, \mathrm{NM} \mathrm{Segerson}^{2}$, and NF Marrouche ${ }^{2}$ \\ ${ }^{1}$ SCI Institute, University of Utah, Salt Lake City, UT, USA \\ ${ }^{2}$ Division of Cardiology, University of Utah, Salt Lake City, UT, USA \\ ${ }^{3}$ CVRTI, University of Utah, Salt Lake City, UT, USA \\ ${ }^{4}$ Bioengineering Department, University of Utah, Salt Lake City, UT, USA \\ ${ }^{5}$ UCAIR, University of Utah, Salt Lake City, UT, USA
}

\begin{abstract}
Magnetic resonance imaging (MRI) based approaches are supporting rapid advances in all phases of the management of atrial fibrillation $(A F)$ patients, especially with the use of contrast agents and novel MRI acquisition techniques. In this report, we summarize briefly some recent advances in our use of MRI for AF management with special focus on the impact of these findings on the modeling and simulation of AF. We summarize results from two clinical studies, one of patients before radio frequency ablation of atrial fibrillation and one after ablation. In pre-ablation patients, significant extent of enhancements in delayed enhancement MRI of the left atrium is predictive of worsened outcome from ablation. The presumed mechanism is the presence of fibrosis in the posterior wall of the left atrium and supports the known finding that patients in chronic atrial fibrillation develop elevated levels of fibrosis. The implications of this finding on modeling of atrial electrical activity are that any such models must include both structural and functional fibrosis if they are to reflect realistic conditions.
\end{abstract}

\section{Introduction}

The overarching goal of our research in atrial fibrillation (AF) is to improve all aspects of the diagnosis, treatment, and management of this condition by means of multimodal medical imaging and image processing. Imaging by means of fluoroscopy, echocardiography, and electroanatomical mapping belong to the current standard of treatment for atrial fibrillation and augment the electrocardiographic findings from standard and Holter ECGs. In addition to the standard imaging modalities, magnetic resonance imaging (MRI) based approaches are supporting rapid advances in all phases of the management of AF pa- tients, especially with the use of contrast agents and novel MRI acquisition techniques. In this report, we summarize briefly some recent advances in our use of MRI for AF management with special focus on the impact of these findings on the modeling and simulation of AF. Our results suggest that heterogeneous composition of atrial tissue, especially in the posterior wall of the left atrium and the atrial septum, is very common in patients with long standing AF and therefore very likely plays an functional role in the establishment and maintenance of AF.

Atrial fibrillation is a growing problem in modern societies with an enormous impact on both short term quality of life and long-term survival. Approximately $0.5 \%$ of people aged 50 to 59 have atrial fibrillation and in the population aged 80 to $89,9 \%$ are afflicted with $\mathrm{AF}$ - and these prevalence are increasing[1]. While many with the condition go untreated, AF is associated with an almost two-fold increase in the risk of mortality. AF patients experience a dramatically increased rate of stroke, from $1.5 \%$ for those aged 50 to 59 years to $23.5 \%$ for those aged between 80 and 89 [2], a risk that, by contrast, decreases with age in the normal population. Treatment of AF represents a significant health-care burden with the annual costs estimated at around 7 billion US dollars [3].

Restoring and maintaining sinus rhythm remains one of the major goals in treating patients with AF. One treatment modality is a combination of DC cardioversion and initiation of antiarrhythmic drugs[4], however, only 40-60\% of the AF population is maintained in regular rhythm one year after such treatment. The treatment itself may also have serious adverse effects[5] and must usually be taken for the lifetime of the patient. The inadequacies of drugbased treatments for AF have long been the major motivation for finding a truly alternative approach to maintain sinus rhythm and suppress AF. 


\subsection{Catheter Ablation of AF}

The past decades have seen significant progress in understanding some of the underlying mechanisms that promote the occurrence of $\mathrm{AF}$ and that encourage its persistence[6] and that knowledge has led to a treatment paradigm with great promise but so far unfulfilled potential. The key finding that led to this therapy was the significant role of the pulmonary veins and the left atrium in initiating and maintaining atrial fibrillation[6,7]. The aim of the resulting therapy has thus become to separate electrically the left atrium from the pulmonary veins. In an effort to increase the penetration of this curative approach, several researchers, including members of our group, have instigated various modifications to the ablation procedure aimed at improving outcome and hence promoting the adoption of the ablation approach by more practicing electrophysiologists[7-13].

Despite the fact that ablation, when successful, offers a complete and final cure, the success rate of ablation in maintaining regular sinus rhythm without the additional use of antiarrhythmic medications still lies at only $40-80 \%$. At least two major obstacles remain that are likely responsible for the wide variation of outcome related to the $\mathrm{AF}$ ablation procedure. The first challenge lies within defining the appropriate candidate for an ablation procedureat present there is no noninvasive means to evaluate the functional status of a potential ablation patient that is adequate to determine the chances of success with that patient. The second ongoing challenge is the assessment of the residual extent and effectiveness of left atrial tissue lesions and eventually scars that are the goal of the procedure. MRI based image analysis approaches provide a potential means to address both challenges.

Triaging patients to the ablation procedure: Defining an accurate means of identifying AF patients who would likely benefit from ablation would greatly improve the success and utility of the procedure. The most promising basis for such a screening lies in specific changes in structure of the atrial tissue that are closely linked to atrial fibrillation. Some of these structural changes occur as a precursor of onset of the disease and some occur as a direct consequence of $\mathrm{AF}$ and further entrench the persistence of the condition-hence the expression that "Atrial fibrillation begets atrial fibrillation"[14]. One functional metric of the state of AF substrate in a patient is the detection of pre-existing low voltage tissue (or fibrosis)[15], which predicts subsequent procedural success with high fidelity. However, pre-existing voltage levels in the heart can only be determined through invasive electrophysiology study of the atrium (electroanatomical mapping).

We have shown that it may be possible to assess noninvasively the location and extent of structural fibrosis within the left atrium prior to ablative treatment by the use of a special technique known as delayed enhancement MRI (DE-MRI) [13,16].

Assessing left atrial tissue damage caused by the ablation procedure: A second major challenge associated with $\mathrm{AF}$ ablation is the assessment of left atrial tissue scar and especially its persistence and extent after recovery. $30-40 \%$ of ablation cases return with recurring $\mathrm{AF}$ and the likely cause is functional (electrophysiological) recovery of the initially ablated tissue. $[10,17,18]$ MRI has the potential to reveal the soft tissue changes that result from ablation of the left atrium and imaging techniques that would allow rapid characterization of the lesions after ablation would have a huge impact on the success of the procedure. Achieving the ability to measure lesion formation in real time or even near real time (within minutes) would improve accuracy of lesion placement and reduce the unwanted recovery of ablated tissue because there would be adequate time to identify and immediately treat locations at which lesion formation in inadequate.

Recently, we and others have developed a high resolution three-dimensional MRI method that makes it possible to perform delayed enhancement imaging of the very thin LA wall in patients[19-21]. In these studies, comparisons between pre-ablation and post-ablation images reveal increased image enhancement, likely due to scar caused by ablation.

\section{Methods}

Image acquisition: We have developed a set of MRI acquisition and image processing methods that are all based on variations of contrasted enhancement through the application of intravenous gadolinium (see other sources for details of the image acquisition techniques[16, 21]). Briefly, acquisition of images consisted of magnetic resonance angiography (MRA) following the initial injection of gadolinium and then delayed enhancement MRI (DEMRI), a technique the involves MRI scans captured 1030 min after gadolinium injection. Typical scan time for the DE-MRI study was 5-9 minutes depending on subject respiration and heart rate. Seventy-three of eighty one patients $(90.1 \%)$ were in normal sinus rhythm during MRI acquisition. Patients who were in atrial fibrillation at the time of clinical presentation were often cardioverted to restore normal sinus rhythm prior to MRI acquisition.

Image processing: In scans performed on patients before treatment, regions of delayed enhancement correspond to elevated levels of fibrosis while elevations in post ablation scans indicate tissue edema (at 24 hours post ablation) and eventually scar formation ( $>3$ months post ablation). Image processing for both was similar and consisted of first manually segmenting using OsiriX (www.osirixviewer.com) and Seg3D (www.seg3d.org the left atrium and then the left atrial wall, then applying statistical thresh- 
olding techniques to highlight regions of elevated enhancement. Initial visualization used a maximum intensity projection (MIP) to assess contrast consistency followed by volume rendering using a ray-cast engine with linear table opacity and application of a Color Look-Up Table (CLUT) mask in order to better differentiate between enhanced and non-enhanced tissue.

We then used the thresholding algorithms to measure the extent of enhancement in each imaging study. For patients evaluated before ablation, we defined three groups based on the extent (percentage of LA myocardium) enhancement such that patients with mild enhancement showed abnormal enhancement in less than $15 \%$ of the LA wall. Moderate enhancement was considered to be between $15 \%$ and $35 \%$ in the LA wall. Extensive enhancement was considered to be greater than $35 \%$ LA wall enhancement.

\section{Results}

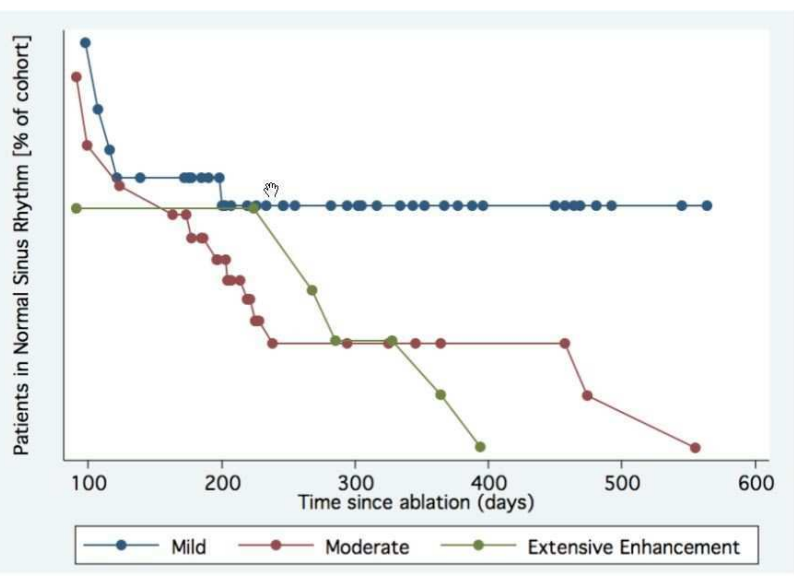

Figure 1. Rate of success of AF ablation for different levels of pre-ablation fibrosis. The three curves correspond to low, moderate, and high levels of pre-ablation enhancement, respectively.n

Using the delayed enhancement methods and image processing described above, for imaging before ablation, we correlated extend of enhancement (fibrosis) with the success of outcome. Figure 1 shows that patients having a moderate or high level of enhancement $(>35 \%)$ showed statistically significantly higher chances of recurrence than patients with only mild levels $(>15 \%)$. Control results for normal subjects showed enhancement levels of $1.7 \% \pm 0.3 \%$.

After ablation, a higher level of enhancement was correlated with successful outcome with $>13 \%$ enhancement a strong positive predictor of successful ablation. In patients with lower levels of post ablation enhancement, the chances of recurrence of the AF were significantly higher.

\section{Discussion and conclusions}

The approaches we have developed and applied to several hundred patients show substantial promise as a noninvasive means of evaluating AF patients both before and after ablation therapy. Applied before therapy, DE-MRI shows the potential to triage patients and establish the suitability of each for subsequent pharmaceutical or ablation treatment. When used after therapy, DE-MRI may provide a means of establishing the success of the therapy and could thus guide further treatments and care. Studies to establish solid backing for these promising results as well as to establish working thresholds and prediction indices are ongoing.

In the context of modeling and simulation, our findings already provide strong indications for the development of clinically relevant computational approaches for evaluating mechanisms and outcomes in patients with AF. Patients with longstanding AF show structural and functional changes in the substrate of the atrial tissues and these same conditions must be part of any clinically relevant simulation model. Out findings suggest that fibrosis must be a structural component of such models and that the altered cell to cell coupling must be reflected in functional aspects of the model. DE-MRI may provide a useful means for creating geometric models of the atria that include the necessary anatomical detail.

\section{Acknowledgements}

The authors gratefully acknowledge the aid of Josh Bertola, Duane Richins and Judy Eldredge who aided with the acquisition of the MRI scans. The authors also acknowledge the computational support and resources provided by the Scientific Computing and Imaging Institute and the NIH NCRR Center for Integrative Biomedical Computing (www.sci.utah.edu/cibc), NIH NCRR Grant No. 5P41RR012553-02. Eugene Kholmovski, Edward DiBella, Dennis Parker and Nassir Marrouche are partially supported by grants from Siemens Medical and Surgivision.

\section{References}

[1] Benjamin EJ, Levy D, Vaziri SM, D’Agostino RB, Belanger AJ, Wolf PA. Independent risk factors for atrial fibrillation in a population-based cohort. the framingham heart study. Jama 1994;271(11):840-844. 0098-7484 (Print) Journal Article Research Support, U.S. Gov't, P.H.S.

[2] Kannel WB, Wolf PA, Benjamin EJ, Levy D. Prevalence, incidence, prognosis, and predisposing conditions for atrial fibrillation: population-based estimates. Am J Cardiol 1998;82(8A):2N-9N.

[3] Coyne KS, Paramore C, Grandy S, Mercader M, Reynolds $\mathrm{M}$, Zimetbaum P. Assessing the direct costs of treating non- 
valvular atrial fibrillation in the united states. Value Health 2006;9(5):348-356. 1098-3015 (Print) Journal Article.

[4] Falk RH. Atrial fibrillation. N Engl J Med 2001; 344(14):1067-1078.

[5] Brodsky MA, Allen BJ, 3rd Walker, C. J., Casey TP, Luckett CR, Henry WL. Amiodarone for maintenance of sinus rhythm after conversion of atrial fibrillation in the setting of a dilated left atrium. Am J Cardiol 1987;60(7):572-575.

[6] Haissaguerre M, Jais P, Shah D, Takahashi A, Hocini M, Quiniou G, Garrigue S, Mouroux AL, Metayer PL, Clementy J. Spontaneous initiation of atrial fibrillation by ectopic beats originating in the pulmonary veins. New Eng J Med 1998;339(10):659-666.

[7] Marrouche N, Dresing T, Cole C, Bash D, Saad E, Balaban K, Pavia S, Schweikert R, Saliba W, Abdul-Karim A, Pisano E, Fanelli R, Tchou P, Natale A. Circular mapping and ablation of the pulmonary vein for treatment of atrial fibrillation: impact of different catheter technologies. J Am Coll Cardiol 2002;40(3):464-474.

[8] Haissaguerre M, Jais P, Shah D, Garrigue S, Takahashi A, Lavergne T, Hocini M, Peng J, Roudaut R, Clementy J. Electrophysiological end point for catheter ablation of atrial fibrillation initiated from multiple pulmonary venous foci. Circ 2000;101(12):1409-1417.

[9] Pappone C, Rosanio S, Oreto G, Tocchi M, Gugliotta F, Vicedomini G, Salvati A, Dicandia C, Mazzone P, Santinelli V, Gulletta S, Chierchia S. Circumferential radiofrequency ablation of pulmonary vein ostia: A new anatomic approach for curing atrial fibrillation. Circulation 2000; 102(21):2619-28.

[10] Marrouche N, Martin D, Wazni O, Gillinov A, Klein A, Bhargava M, Saad E, Bash D, Yamada H, Jaber W, Schweikert R, Tchou P, Abdul-Karim A, Saliba W, Natale A. Phased-array intracardiac echocardiography monitoring during pulmonary vein isolation in patients with atrial fibrillation: impact on outcome and complications. Circ 2003; 107(21):2710-2716.

[11] Chen M, Marrouche N, Khaykin Y, Gillinov A, Wazni O, Martin D, Rossillo A, Verma A, Cummings J, Erciyes D, Saad E, Bhargava M, Bash D, Schweikert R, Burkhardt D, Williams-Andrews M, Perez-Lugones A, Abdul-Karim A, Saliba W, Natale A. Pulmonary vein isolation for the treatment of atrial fibrillation in patients with impaired systolic function. J Am Coll Cardiol March 2004;43(6):1004-1009.

[12] Gillinov A, McCarthy P, Blackstone E, Rajeswaran J, Pettersson G, Sabik J, Svensson L, Cosgrove D, Hill K, Gonzalez-Stawinski G, Marrouche N, Natale A. Surgical ablation of atrial fibrillation with bipolar radiofrequency as the primary modality. J Thorac Cardiovasc Surg June 2005; 129(6):1322-1329.

[13] Badger T, Oakes R, Fish N, Blauer J, McGann C, MacLeod R, Marrouche N. New MRI method to detect abnormal electrical tissue using delayed enhancement MRI: Correlation with electroanatomic voltage maps. In American College of Cardiology. Chicago, Illinois, 2008; to appear.

[14] Wijffels MC, Kirchhof CJ, Dorland R, Allessie MA. Atrial fibrillation begets atrial fibrillation. A study in awake chronically instrumented goats. Circ 1995;92(7):1954-1968.
0009-7322 (Print) Journal Article Research Support, NonU.S. Gov't.

[15] Verma A, Kilicaslan F, Pisano E, Marrouche N, Fanelli R, Brachmann J, Geunther J, Potenza D, Martin D, Cummings J, Burkhardt J, Saliba W, Schweikert R, Natale A. Response of atrial fibrillation to pulmonary vein antrum isolation is directly related to resumption and delay of pulmonary vein conduction. Circ 2005;112(5):627-635.

[16] Oakes R, Badger T, Khoumovski E, Segerson N, Burgon N, Fish E, Blauer J, Rao S, DiBella E, , Daccarett NAM, Winfelder J, McGann C, Parker D, MacLeod R, Marrouche $\mathrm{N}$. Detection and quantification of left atrial structural remodeling using delayed enhancement MRI in patients with atrial fibrillation. Circ 2008; (in review).

[17] Dilaveris PE, Gialafos EJ, Andrikopoulos GK, Richter DJ, Papanikolaou V, Poralis K, Gialafos JE. Clinical and electrocardiographic predictors of recurrent atrial fibrillation. Pacing Clin Electrophysiol 2000;23(3):352-8.

[18] Ellis K, Wazni O, Marrouche N, Martin D, Gillinov M, McCarthy P, Saad E, Bhargava M, Schweikert R, Saliba W, Bash D, Rossillo A, Erciyes D, Tchou P, Natale A. Incidence of atrial fibrillation post-cavotricuspid isthmus ablation in patients with typical atrial flutter: Left-atrial size as an independent predictor of atrial fibrillation recurrence. J Cardiovasc Electrophysiol June 2007; (in press).

[19] Peters DC, Wylie JV, Hauser TH, Kissinger KV, Botnar RM, Essebag V, Josephson ME, Manning WJ. Detection of pulmonary vein and left atrial scar after catheter ablation with three-dimensional navigator-gated delayed enhancement MR imaging: initial experience. Radiology 2007; 243(3):690-5.

[20] Reddy VY, Schmidt EJ, Holmvang G, Fung M. Arrhythmia recurrence after atrial fibrillation ablation: can magnetic resonance imaging identify gaps in atrial ablation lines? J Cardiovasc Electrophysiol 2008;19(4):434-7.

[21] McGann C, Kholmovski E, Oakes R, Blauer J, Daccarett M, Segerson N, Airey K, Akoum N, Fish E, Badger T, DiBella E, Parker D, MacLeod R, Marrouche N. New magnetic resonance imaging based method to define extent of left atrial wall injury after the ablation of atrial fibrillation. J Am Coll Cardiol 2008;(in press).

Address for correspondence:

Rob MacLeod

University of Utah

Scientific Computing and Imaging (SCI) Institute

72 South Central Campus Dr., 3750 WEB

E-mail addressmacleod@cvrti.utah.edu 